\title{
Trainee caseload correlates with ERCP success rates but not with procedure-related complications: results from a prospective study (the QUASIE cohort)
}

Authors

Institutions
Theodor Voiosu ${ }^{1}$, Andreea Bengus ${ }^{1}$, Andrei Voiosu' ${ }^{1}$, Mihai Rimbas ${ }^{1}$, Alina Zlate ${ }^{1}$, Andrei Haidar ${ }^{1}$, Cristian Baicus ${ }^{2}$, Bogdan Mateescu ${ }^{1}$

${ }^{1}$ Gastroenterology Department, Colentina Clinical Hospital, Bucharest, Romania

${ }^{2}$ Internal Medicine Department, Colentina Clinical Hospital, Bucharest, Romania submitted 7. October 2015 accepted after revision 19. January 2016

\section{Bibliography}

Dol http://dx.doi.org/

10.1055/s-0042-102248

Published online: 30.3.2016

Endoscopy International Open

2016; 04: E409-E414

(c) Georg Thieme Verlag KG

Stuttgart · New York

E-ISSN 2196-9736

\section{Corresponding author}

\section{Theodor Voiosu, MD, PhD}

Gastroenterology Department Colentina Clinical Hospital 19-21 Stefan cel Mare Boulevard Bucharest

Romania

Fax: +40-21-3180604

theodor.voiosu@gmail.com
Background and study aim: Endoscopy society guidelines recommend a minimum of 200 cases for endoscopic retrograde cholangiopancreatography (ERCP) trainees in order to ensure competency and quality standards. However, there are few data regarding procedure-related complication rates and added risk for patients during this learning process. We aimed to evaluate the correlation between trainee caseload and procedureand patient-related outcomes in an ERCP training program, and to assess the risk factors for ERCP failure and complications.

Patients and methods: We conducted a prospective study of all procedures performed in the ERCP training program at Colentina Clinical Hospital, Bucharest, Romania. Relevant data for each procedure (diagnosis, cannulation method, outcome, and complications during the following 30 days) as well as operator experience were documented. Univariable and multivariable analysis of the risk factors for ERCP failure and complications was done by analyzing the procedures completed by expert and trainee endoscopists during the study period.

\section{Introduction}

$\nabla$

Endoscopic retrograde cholangiography (ERCP) has evolved from a diagnostic to mainly a therapeutic procedure during the past decades, allowing the minimally invasive treatment of both benign and malignant diseases of the bile ducts and pancreas. Although ERCP is generally considered a safe procedure, complications including postERCP pancreatitis, bleeding, and perforation can occur in up to $10 \%$ of cases, with an associated mortality rate of about $1 \%[1,2]$.

Data from large nationwide registries have shown that more experienced endoscopists with high case volumes have higher success rates and fewer procedure-related complications than do less experienced endoscopists with low case volumes [3,
Results: The analysis included 534 ERCPs performed by 1 expert and 3 supervised trainees during a 12-month period. Technical success rates were comparable in the trainee and expert groups, and no statistically significant difference was found between the two groups with regard to procedure-related complications and mortality. The more experienced trainees had a better chance of successfully completing a procedure (odds ratio of 1.1 for each additional 10 ERCPs performed), but post-ERCP complications were unrelated to individual trainee caseloads on multivariable analysis.

Conclusion: The ERCP technical success rate increases with trainee experience, reflecting the learning curve of individual operators. However, the complication rates are similar across different levels of operator experience, indicating that ERCPs performed by supervised trainees imply no additional risk for patients.

4]. These results are supported by earlier studies from teaching hospitals showing that most trainees achieve satisfactory levels of competency after performing more than 150 to 200 procedures [5,6].

Based on these observations, competency in ERCP is now granted based on specific requirements, such as completing a minimum of 200 procedures and achieving an overall biliary cannulation rate of at least $85 \%[7,8]$. However, currently there are limited data available on the additional risk for complications when procedures are performed during training programs. We aimed to explore the relationship between the learning curve of endoscopy fellows and procedure-related complications in a training program setting. 


\section{Patients and methods}

$\nabla$

\section{Patients}

We conducted a prospective study of all patients undergoing ERCP in our unit during a 12-month period from January 2014 to January 2015. Each patient's age, sex, and indication for ERCP were documented. Information about the type and level of difficulty of each procedure according to the proposed American Society for Gastrointestinal Endoscopy (ASGE) scale ( $\bullet$ Table 1 [9]), the patient's papillary anatomy (native papilla/previous sphincterotomy), and the cannulation method (guidewire, contrast injection, precut sphincterotomy) was provided by the attending endoscopist through a standard report form. Patients were followed for up to 30 days after the procedure via their attending physician or primary caregiver, and procedure-related complications (bleeding, post-ERCP pancreatitis, perforation, cholangitis, and death) were documented. The study design was approved by the local ethics committee, and all patients signed a standard informed consent form before undergoing the endoscopic procedure.

\section{Training program}

The standard procedure in our unit is first to attempt cannulation of a native papilla by the guidewire technique and in case of failure subsequently to use other techniques (contrast injection, precut sphincterotomy) to obtain selective cannulation of the desired duct. In this study, there was no time limit for attempted cannulation; however, if the procedure was performed by a trainee, a maximum of 10 minutes was allowed for cannulation before the expert operator took over the procedure. If at the end of the 10-minute interval the cannulation was unsuccessful, the expert could decide either to take over and continue the procedure or to repeat the procedure after an interval of 24 to 48 hours. This decision was based in each case on the expert's clinical judgment. Only operators who performed at least 20 procedures during the study period according to this protocol were included in the final analysis. A 100-mg diclofenac suppository was administered intrarectally to all patients after their procedures in order to minimize the risk for post-ERCP pancreatitis.

\section{Outcome measures}

The outcome measures for our study were successful cannulation of the desired duct, rates of technical success (overall success of the procedure), and procedure-related complication rates. Technical success was defined as completion of the intended procedure (e.g., stent insertion, stone extraction). Procedure-related complications (post-ERCP pancreatitis, cholangitis, bleeding, and 30-day mortality) were documented and graded as mild, moder-

Table 1 Scale of difficulty for biliopancreatic procedures.
Level of difficulty*
\begin{tabular}{cl} 
I & Deep CBD / PD cannulation, stent replacement \\
II & Distal CBD stricture, PD stenting, stone extraction $<10 \mathrm{~mm}$ \\
\hline III & $\begin{array}{l}\text { Stone extraction }>10 \mathrm{~mm}, \text { PD stone extraction }<5 \mathrm{~mm} \text {, papilla } \\
\text { minor cannulation in patient with pancreas divisum, hilar stricture }\end{array}$ \\
IV & $\begin{array}{l}\text { Whipple / Roux-en-Y anatomy, migrated PD stent, intraductal } \\
\text { imaging }\end{array}$
\end{tabular}

CBD, common bile duct; PD, pancreatic duct.

* Addition of 1 point for previously failed procedure, procedure conducted outside office hours, and procedure conducted in patients younger than 5 years of age. ate, or severe according to the criteria proposed by Cotton et al. [10].

\section{Learning curve and procedure outcome}

The caseload of each trainee was divided into blocks of 10 successive procedures in order to evaluate the role of the learning curve in relation to the overall success rate of the procedures and the occurrence of complications. The number of cases completed before the study period was available and taken into account for each trainee. A case in which an expert took over from a trainee during any part of the procedure was excluded from the final analysis but was included in the trainee's total caseload. We compared the adverse event rates and technical success rates by blocks of procedures for the entire subgroup of cases conducted by trainees in order to assess the relationship between trainee experience and procedure-related outcomes.

\section{Sample size estimation}

Based on an estimated $90 \%$ technical success rate in the expert group of cases, we calculated that a total of 438 procedures (219 in each group) would be required to detect a $10 \%$ difference between the expert and trainee groups at an alpha of 0.05 and a beta of 0.200 . This sample size would also allow the detection of an increase in the overall adverse event rate from an estimated $10 \%$ in the expert group to $20 \%$ in the trainee group at a power of $80 \%$ and a one-sided $P$ value of less than 0.05 .

\section{Data analysis}

Data were recorded and analyzed with SPSS for Windows, Version 16.0 (SPSS Inc., Chicago, Illinois, USA). Results were reported as mean and standard deviation for variables with a normal distribution, and as median, minimum, and maximum for variables with a non-normal distribution. Univariable analysis was conducted with the chi-squared test for nominal variables, with nonparametric tests (Mann - Whitney $U$ test, Kruskall - Wallis test) for variables with a non-normal distribution, and with Student's $t$ test for variables with a normal distribution.

Multivariable analysis by logistic regression was used to analyze risk factors for technical success as well as for procedure-related complications. For the regression model, the traditional risk factors for procedure-related complications were selected as covariates before initiation of the study: sex, age, bilirubin levels, technical difficulty of the procedure (ASGE scale), method of cannulation, and operator experience (expert vs. trainee). Two-sided hypothesis testing was used, with a $P$ value of less than 0.05 considered statistically significant.

\section{Results \\ $\nabla$}

From January 31, 2014, to January 31, 2015, a total of 581 consecutive procedures were performed in the endoscopy department at Colentina Clinical Hospital. A total of 534 ERCPs conducted in 476 patients (range $1-4$ procedures per patient) by 4 endoscopists (3 trainees starting with an average experience of 60 ERCPs and 1 expert operator with experience of more than 3000 ERCPs) were included in the final analysis ( $\bullet$ Fig. 1 ). The characteristics of the patients included in the study are detailed in $\bullet$ Table 2. The trainees performed 283 procedures (53\%) and an expert endoscopist performed 251 procedures (47\%). The level of difficulty of the caseloads according to the ASGE scale is detailed in 0 Table 3. 


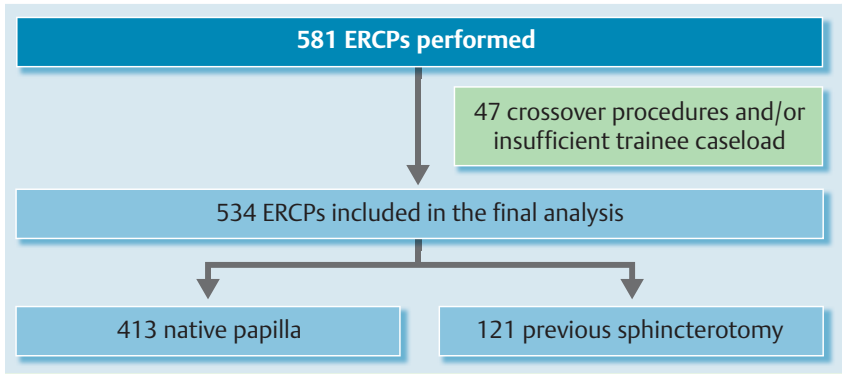

Fig. 1 Flowchart of the procedures performed during the study period. ERCP, endoscopic retrograde cholangiopancreatography.
Table 3 Comparison of trainee and expert caseloads in regard to difficulty.

\begin{tabular}{|lccc|} 
& Trainees & Expert & P value \\
\hline ASGE level of difficulty & & & \\
\hline Level I & 20 & 27 & \\
\hline Level II & 221 & 195 & $0.08^{1}$ \\
\hline Level III & 41 & 28 & \\
\hline Level IV & 1 & 1 & \\
\hline Patient with native papilla & $210 / 283$ & $203 / 251$ & $0.07^{2}$ \\
\hline
\end{tabular}

ASGE, American Society for Gastrointestinal Endoscopy.

${ }^{1}$ Not significant with Mann - Whitney $U$ test.

${ }^{2}$ Not significant with chi-squared test.

and type of procedure-related complications across the entire study group ( $\odot$ Table 4 ) and in the patients with a native papilla (० Table 5).

\section{Risk factors for procedural failure and complications}

On multivariable analysis, female sex was associated with an increased risk for any procedure-related adverse event (odds ratio [OR] 2.2, 95\%CI 1.1-4.5). In the patients with a native papilla, increases in the bilirubin level were associated with a higher risk for post-ERCP cholangitis and a higher risk for technical failure, with ORs of 1.14 (95\%CI $1.03-1.28)$ and 1.04 (95\%CI $1.01-1.06)$, respectively, for each increase in the serum bilirubin level of 1 $\mathrm{mg} / \mathrm{dL}(\bullet$ Table 6$)$. No risk factors for post-ERCP pancreatitis were identified on multivariable analysis, although a trend toward more post-ERCP pancreatitis in female patients was noted $(P=0.1)$.

\section{Trainee caseload and procedure outcome}

On multivariable analysis, after adjustment for age and sex of the patients and grade of difficulty of the procedures, the procedures performed by trainees with a higher caseload were more likely to succeed than those performed by less experienced trainees (OR $1.1,95 \%$ CI $1.01-1.19$ for each additional 10 procedures performed). However, no statistically significant difference in the adverse event rates according to trainee experience was found on multivariable analysis (OR 1.04, 95\%CI $0.93-1.17$ for each additional 10 procedures performed) ( $\bullet$ Fig. $2, \otimes$ Fig. 3, $\odot$ Table 7$)$. the expert group and a $91.8 \%$ success rate in the trainee group $(P=0.8$, chi-squared test $)$.

In regard to cannulation technique, most procedures were performed with the guidewire technique ( $83 \%)$, followed by precut sphincterotomy (11\%) and contrast-guided cannulation (6\%); significantly more precut procedures were performed in the expert group ( 32 vs. $7, P<0.001$, chi-squared test).

No significant differences were found between the expert and trainee groups regarding procedural technical success and rate

\section{Discussion}

The main finding of our study is that although technical success increases with increased trainee caseload, there seems to be no correlation between trainee caseload and procedure-related complications. Also, our data suggest that within a training pro-

\begin{tabular}{|lllll|}
\hline Characteristic & Total & Trainees & Expert & $P$ value \\
\hline Female sex, $\mathrm{n}(\%)$ & $271(50.7)$ & $133(47)$ & $138(55)$ & 0.06 \\
\hline Age, mean (SD), $\mathrm{y}$ & $64(15.3)$ & $65.5(14.7)$ & $62.2(16.1)$ & $0.017^{*}$ \\
\hline Native papilla, $\mathrm{n}(\%)$ & $413(77.3)$ & $210(74.2)$ & $203(80.8)$ & \\
\hline Indication for ERCP, $\mathrm{n}(\%)$ & & & \\
\hline CBD stones & $325(60.8)$ & $164(57.9)$ & $161(64.1)$ & 0.34 \\
\hline Malignant CBD stricture & $121(22.6)$ & $70(24.7)$ & $51(20.3)$ & $39(15.5)$ \\
\hline Other & $88(16.5)$ & $49(17.3)$ & $163(64.9)$ & 0.07 \\
\hline Increased bilirubin $(>1 \mathrm{mg} / \mathrm{dL})$ & $370(69.2)$ & $207(73.1)$ & & \\
\hline
\end{tabular}

Table 2 Characteristics of patients included in a study comparing outcomes of ERCP performed by trainess with outcomes of ERCP performed by an expert.

$\mathrm{SD}$, standard deviation; ERCP, endoscopic retrograde cholangiopancreatography; $\mathrm{CBD}$, common bile duct.

* Statistically significant with Student's $t$ test. 
Table 4 Comparison of outcome measures in the entire study group: procedures completed by trainees vs. those completed by the expert.

\begin{tabular}{|lcccl}
\hline Outcome measure & Trainees, $\mathbf{n}(\%)[\mathbf{9 5} \% \mathbf{C l}]$ & Expert, $\mathbf{n}(\%)[95 \% \mathbf{C l}]$ & RR [95\%Cl] & P value \\
\hline Technical success & $220(77.7)[72.8-82.8]$ & $193(76.8)[71.2-82.0]$ & $1.01[0.9-1.1]$ & 0.75 \\
\hline Adverse events & $25(8.8)[5.8-12.8]$ & $17(6.7)[4.0-10.6]$ & $1.3[0.7-2.4]$ & 0.42 \\
\hline PEP & $16(5.6)[3.3-9.0]$ & $15(5.9)[3.4-9.7]$ & $0.95[0.5-1.9]$ & 0.88 \\
\hline Cholangitis & $7(2.4)[1.0-5.0]$ & $3(1.1)[0.2-3.4]$ & $2.1[0.5-8.0]$ & 0.34 \\
\hline Mortality & $4(1.4)[0.4-3.6]$ & $1(0.4)[0.1-2.2]$ & $3.6[0.4-31.6]$ & 0.37 \\
\hline Bleeding & $4(1.4)[0.4-3.6]$ & $9(3.5)[1.4-6.2]$ & $0.4[0.1-1.4]$ & 0.24
\end{tabular}

$\mathrm{Cl}$, confidence interval; RR, relative risk; PEP, post-endoscopic retrograde cholangiopancreatography (ERCP) pancreatitis.

Table 5 Comparison of outcome measures in the patients with a native papilla: procedures completed by trainees vs. those completed by the expert.

\begin{tabular}{|c|c|c|c|c|}
\hline Outcome measure & Trainees, n (\%) [95\%Cl] & Expert, n (\%) [95\%Cl] & $\mathrm{RR}[95 \% \mathrm{Cl}]$ & $P$ value \\
\hline Successful cannulation & $182(86.6)$ & $170(83)$ & & 0.43 \\
\hline Technical success & $162(77.1)[71.6-83.3]$ & $150(73.8)[70.3-82.4]$ & $1.0[0.9-1.1]$ & 0.81 \\
\hline Adverse event & $20(9.5)[6.0-14.5]$ & $13(6.4)[3.5-10.8]$ & $1.5[0.8-2.9]$ & 0.27 \\
\hline PEP & $14(6.6)[3.7-11.0]$ & $13(6.4)[3.5-10.7]$ & $1.0[0.5-2.2]$ & 1 \\
\hline Cholangitis & $4(1.9)[0.5-4.9]$ & $1(0.05)[0.0-1.8]$ & $8.7[0.5-160.6]$ & 0.37 \\
\hline Bleeding & $4(1.9)[0.5-4.8]$ & $9(4.4)[2.1-8.3]$ & $0.43[0.13-1.37]$ & 0.16 \\
\hline Mortality & $3(1.4)[0.3-4.1]$ & $0(0)[0.0-1.8]$ & $6.8[0.3-130.2]$ & 0.62 \\
\hline
\end{tabular}

$\mathrm{Cl}$, confidence interval; RR, relative risk; PEP, post-endoscopic retrograde cholangiopancreatography (ERCP) pancreatitis.

gram, quality standards (i.e., successful cannulation, technical success) can be met with no additional risk for patients undergoing procedures. These are salient findings because patient safety during training programs in endoscopy is of paramount importance. Currently, however, few data are available concerning procedure outcomes and complication rates in this setting [11].

Learning curve studies in ERCP trainees show that approximately 200 procedures are required before a trainee can consistently achieve technical success $[12,13]$. Furthermore, a higher volume of procedures per endoscopist $[4,14]$ and per endoscopy service [15] seems to correlate with increased procedural success rates and fewer complications. We aimed to evaluate whether there might be a similar correlation between the learning curve for ERCP and procedure-related complications.

All the trainees in our study had previous experience performing ERCP, with an average caseload of 60 procedures before the start of the study period. The technical success rates and complication rates of the individual trainees did not differ significantly, but the technical success rates significantly improved as the volume of procedures performed increased.

On multivariable analysis, a higher trainee caseload correlated with improved technical success rates, a finding consistent with data from previous studies [4-7]. However, the risk for procedure-related complications was no greater when procedures were performed by a trainee than when they were performed by an expert, regardless of the trainee caseload or procedure difficulty. Also, the overall cannulation and technical success rates were similar in the two study groups and in accordance with proposed quality standards for ERCP [16]. This finding is extremely important because it confirms that within the confines of a wellstructured training program, patients undergoing ERCPs performed by trainee endoscopists are not subjected to any additional risk.

An interesting finding is that the rates of successful cannulation were similar in the trainee group (86.6\%) and the expert group $(83 \%)$, with a per-patient successful cannulation rate of $92.3 \%$.
Given the relatively limited number of procedures analyzed and the small number of endoscopists in our study, these results require further validation in randomized controlled trials. However, it seems likely that supervised trainees can achieve the quality standards currently proposed (i.e., a cannulation rate of approximately $85 \%$ ).

An important limitation of our study is the lack of randomization of procedures between the trainees and the expert. Although our comparison shows that the two groups were very similar in terms of patient age, diagnoses, and procedure complexity, there was still a high risk for selection bias before the procedures. This theoretical selection bias and other potential confounding factors could also account for the relatively low cannulation rate and technical success rate of the expert endoscopist compared with previously reported data $[1-4]$. We used multivariable analysis to account for traditional risk factors for complications (e.g., age, bilirubin levels, failed cannulation, malignant strictures) in an attempt to minimize the risk for such bias.

Another potential limitation is the exclusion of cases in which more than one operator was involved in completing the procedure (crossover cases) from the final analysis. In the setting of a training program, it is inevitable that some procedures will have to be completed by experts, but evaluating and attributing the additional risk for the patient is very difficult in such cases because the role of the expert can vary considerably between interventions. In our cohort, only 47 procedures $(8.1 \%)$ were excluded

Table 6 Risk factors for various procedure-related outcomes obtained with multivariate analysis (logistic regression).

\begin{tabular}{|llll|}
\hline Factor & Outcome & OR & $\mathbf{9 5 \% C l}$ \\
\hline Female sex & Any adverse event & 2.2 & $1.1-4.5$ \\
\hline Bilirubin level & Cholangitis & 1.14 & $1.03-1.28^{*}$ \\
\hline Bilirubin level & Technical failure & 1.04 & $1.01-1.06^{*}$ \\
\hline
\end{tabular}

$\mathrm{OR}$, odds ratio; $\mathrm{Cl}$, confidence interval.

* Relative risk for every increase in serum bilirubin of $1 \mathrm{mg} / \mathrm{dL}$. 

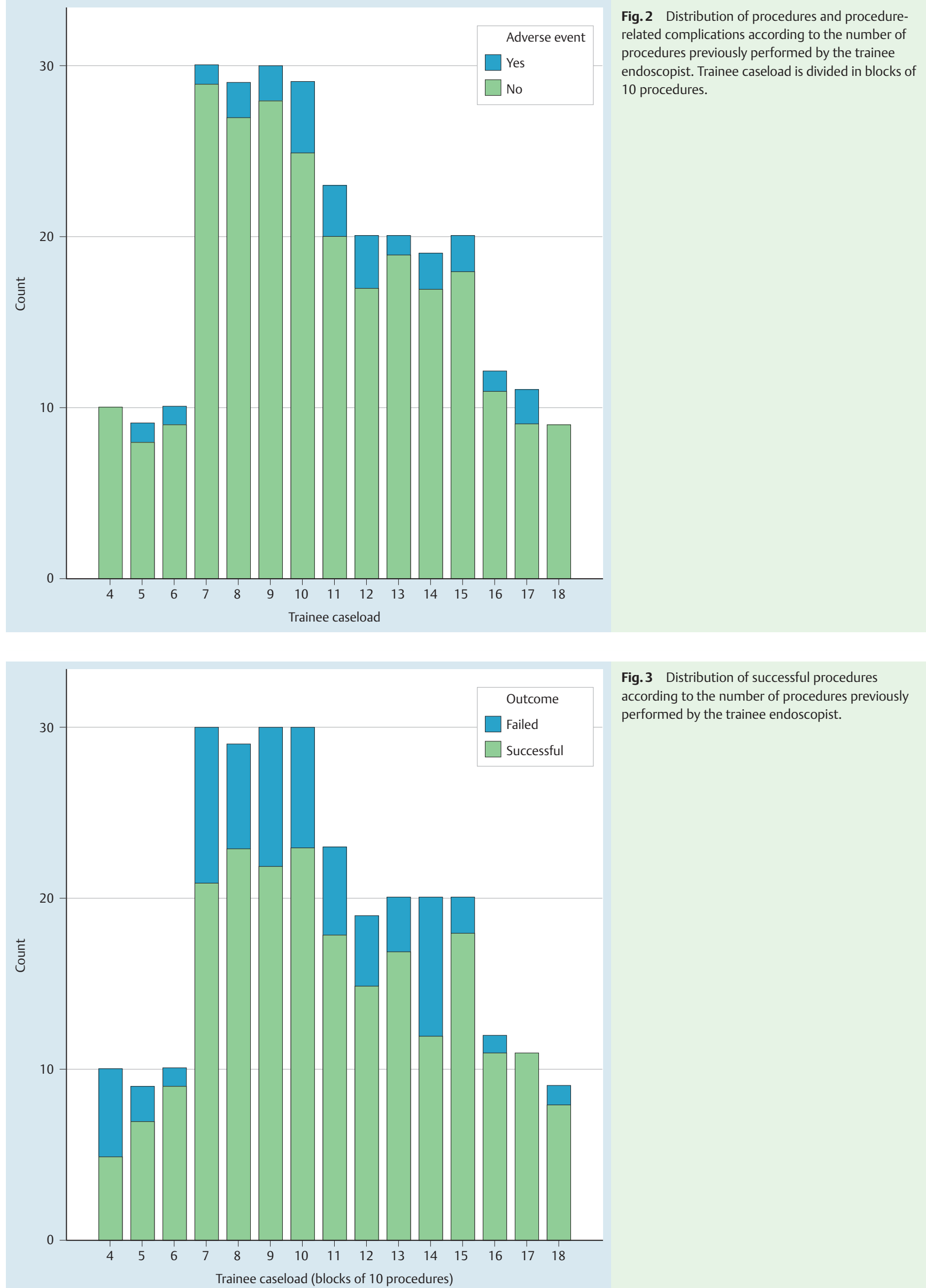

Fig. 3 Distribution of successful procedures according to the number of procedures previously performed by the trainee endoscopist. 
Table 7 Trainee caseload cross-tabulation.

\begin{tabular}{|c|c|c|c|c|c|c|c|c|c|c|c|c|c|c|c|c|c|}
\hline \multirow[t]{2}{*}{ Count } & & \multicolumn{15}{|c|}{ Trainee caseload } & \multirow[t]{2}{*}{ Total } \\
\hline & & 4 & 5 & 6 & 7 & 8 & 9 & 10 & 11 & 12 & 13 & 14 & 15 & 16 & 17 & 18 & \\
\hline \multirow{2}{*}{$\begin{array}{l}\text { Adverse } \\
\text { event }\end{array}$} & No & 10 & 8 & 9 & 29 & 27 & 28 & 25 & 20 & 17 & 19 & 17 & 18 & 11 & 9 & 9 & 256 \\
\hline & Yes & 0 & 1 & 1 & 1 & 2 & 2 & 4 & 3 & 3 & 1 & 2 & 2 & 1 & 2 & 0 & 25 \\
\hline Total & & 10 & 9 & 10 & 30 & 29 & 30 & 29 & 23 & 20 & 20 & 19 & 20 & 12 & 11 & 9 & 281 \\
\hline \multirow[t]{2}{*}{ Outcome } & Failed & 5 & 2 & 1 & 9 & 6 & 8 & 7 & 5 & 4 & 3 & 8 & 2 & 1 & 0 & 1 & 62 \\
\hline & Successful & 5 & 7 & 9 & 21 & 23 & 22 & 23 & 18 & 15 & 17 & 12 & 18 & 11 & 11 & 8 & 220 \\
\hline Total & & 10 & 9 & 10 & 30 & 29 & 30 & 30 & 23 & 19 & 20 & 20 & 20 & 12 & 11 & 9 & 282 \\
\hline
\end{tabular}

from the final analysis, and we did not detect an increased complication rate in these cases, with an overall adverse event rate of $8.5 \%$ and no fatal cases reported in this particular subgroup.

With regard to post-ERCP complications, our data suggest that the risk for complications is no greater in procedures carried out by a trainee than in those carried out by an experienced endoscopist. However, given the number of procedures included in the analysis, we acknowledge that our study may not have been adequately powered to detect potentially significant differences in regard to adverse events with a low prevalence. This issue needs to be explored further, preferably in a multicenter trial setting.

Despite these limitations, we believe that our study provides important evidence toward a better understanding of ERCP and ways to improve teaching. The fact that the procedures carried out by supervised trainees carried no additional risk for the patients while technical success rates remained satisfactory is an important finding that helps shed light on an area where it is sorely needed - procedure outcome and patient safety in endoscopy teaching programs.

\section{Competing interests: None}

\section{References}

1 Suissa A, Yassin K, Lavy A et al. Outcome and early complications of ERCP: a prospective single center study. Hepatogastroenterology 2005; 52: $352-355$

2 Christensen M, Matzen P, Schulze $S$ et al. Complications of ERCP: a prospective study. Gastrointest Endosc 2004; 60: 721 - 731

3 Ekkelenkamp VE, de Man RA, Ter Borg F. Prospective evaluation of ERCP performance: results of a nationwide quality registry. Endoscopy 2015; 47: 503-507

4 Kapral C, Duller C, Wewalka F et al. Case volume and outcome of endoscopic retrograde cholangiopancreatography: results of a nationwide Austrian benchmarking project. Endoscopy 2008; 40: 625-630
5 Jowell PS, Baillie J, Branch MS et al. Quantitative assessment of procedural competence. A prospective study of training in endoscopic retrograde cholangiopancreatography. Ann Intern Med 1996; 125: $983-$ 989

6 Verma D, Gostout CJ, Petersen BT et al. Establishing a true assessment of endoscopic competence in ERCP during training and beyond: a singleoperator learning curve for deep biliary cannulation in patients with native papillary anatomy. Gastrointest Endosc 2007; 65: 394-400

7 Chutkan RK, Ahmad AS, Cohen J et al. ERCP core curriculum. Gastrointest Endosc 2006; 63: 361 - 376

8 Ang TL, Cheng J, Khor JL et al. Guideline on training and credentialing in endoscopic retrograde cholangiopancreatography. Singapore Med J 2011; 52: 654-657

9 Cotton PB, Eisen G, Romagnuolo J. Grading the complexity of endoscopic procedures: results of an ASGE working party. Gastrointest Endosc 2011; 73: 868-874

10 Cotton PB, Lehman G, Vennes J et al. Endoscopic sphincterotomy complications and their management: an attempt at consensus. Gastrointest Endosc 1997; 37: 383-393

11 Jowell PS. Endoscopic retrograde cholangiopancreatography: toward a better understanding of competence. Endoscopy 1999; 31: 755 - 757

12 Verma D, Gostout CJ, Petersen BT et al. Establishing a true assessment of endoscopic competence in ERCP during training and beyond: a singleoperator learning curve for deep biliary cannulation in patients with native papillary anatomy. Gastrointest Endosc 2007; 65: 394-400

13 Ekkelenkamp VE, Koch AD, Rauws EA et al. Competence development in ERCP: the learning curve of novice trainees. Endoscopy 2014; 46: 949-955

14 Katsinelos P, Mimidis K, Paroutoglou G et al. Needleknife papillotomy: a safe and effective technique in experienced hands. Hepatogastroenterology 2004; 51: 349-352

15 Varadarajulu S, Kilgore ML, Wilcox CM. Relationship among hospital ERCP volume, length of stay, and technical outcomes. Gastrointest Endosc 2006; 64: 338-347

16 Baron TH, Petersen BT, Mergener $K$ et al. Quality indicators for endoscopic retrograde cholangiopancreatography. Am J Gastroenterol 2006; 101: $892-897$ 